\title{
Methods for Structuring and Prototyping on a Nanoscale Using a DualBeam
}

\author{
F. Morrissey ${ }^{*}$, S. Reyntjens ${ }^{* *}$, K. Nakahara ${ }^{* * *}$, C. Jiao* \\ *FEI Company, The Aztec Centre, Bristol BS32 4TD, UK \\ ***FE Company, Building AAE, Achtseweg Noord, PO Box 80066, Eindhoven, The Netherlands \\ ${ }^{* * *}$ FEI Company, 5350 Dawson Creek Drive, Hillsboro OR 97124, USA
}

DualBeam is a unique combination of a focused ion beam (FIB) and a scanning electron microscope (SEM) that is becoming a popular tool for materials science and nanotechnology. FIB has been used for some time to perform surgery on microchips, and more recently, to prepare site-specific samples for techniques such as TEM. With the advent of DualBeam, it has become easier to characterize very small structures made with the FIB non-destructively by utilizing the SEM for imaging. Improved techniques and software have honed the process of milling and viewing with the electron beam, even allowing the two beams to interact, so that nanoscale fabrication and prototyping can take place in real-time. This immediate feedback means that prototyping can quickly proceed to a result, making the nano-world much more accessible. In this paper a number of methods of utilizing the DualBeam for structuring and prototyping on the nanoscale will be discussed using practical examples for illustration.

One of the primary requirements for a nanoscale prototyping tool is that it can produce coherent structures, on demand, at the scale required and have sufficient imaging resolution to determine the outcome. Figure 1 shows a FIB milled cross in a chromium film on a glass substrate with minimum milled features of $10 \mathrm{~nm}$ or less. Once such a feature is produced, the next requirement is often to repeat it, to produce a regular array of features. To do this, a way to step and repeat over an area much larger than the original feature is required. Figures 2, 3a, and 4 show three different methods of achieving this. In figure 2, a complex pattern has been produced using the DualBeam's patterning engine. A script has then been used to mill this pattern over a field of view and then make a very accurate stage movement to repeat this over several fields of view. A more accurate way of repeating the pattern over a relatively large area is to use the electronic beamshift of the system to move the pattern rather than moving the stage. The beamshift on the DualBeam has a large range but can achieve very fine movements. Figure 3 a shows an optical grating milled over a $100 \mu \mathrm{m}$ field using the beamshift method, figure $3 \mathrm{~b}$ shows a cross section through part of the same grating. To minimize placement inaccuracies over a large field where stage movements are involved, recognition marks are used to identify and accurately place the beam on the sample using a combination of stage moves and beamshift. Using this method, Figure 4 shows part of a grating, several hundred microns long, milled in GaAs - the individual lines are $100 \mathrm{~nm}$ wide.

Sometimes only one very small milled feature is required but it has to be placed very accurately with respect to some other feature. These indexing features are often very small and very sensitive to the ion beam. So, ideally, the electron beam should be used to locate the feature and steer the ion beam to mill in exactly the correct position. Figure 5 illustrates a method where this has been achieved with a DualBeam over multiple sites, to an accuracy of $<20 \mathrm{~nm}$. The central right-angled feature represents the indexing feature, found by the electron beam alone. The electron beam then places the second rightangled feature in the bottom left of the image for the ion beam to mill - this serves as an alignment point. The system then measures the distance from the indexing feature to the alignment point and accurately places the required milled feature (the cross in this image). Thus DualBeam can accurately place point specific, nanoscale mills using only the electron beam for navigation and pattern placement. 

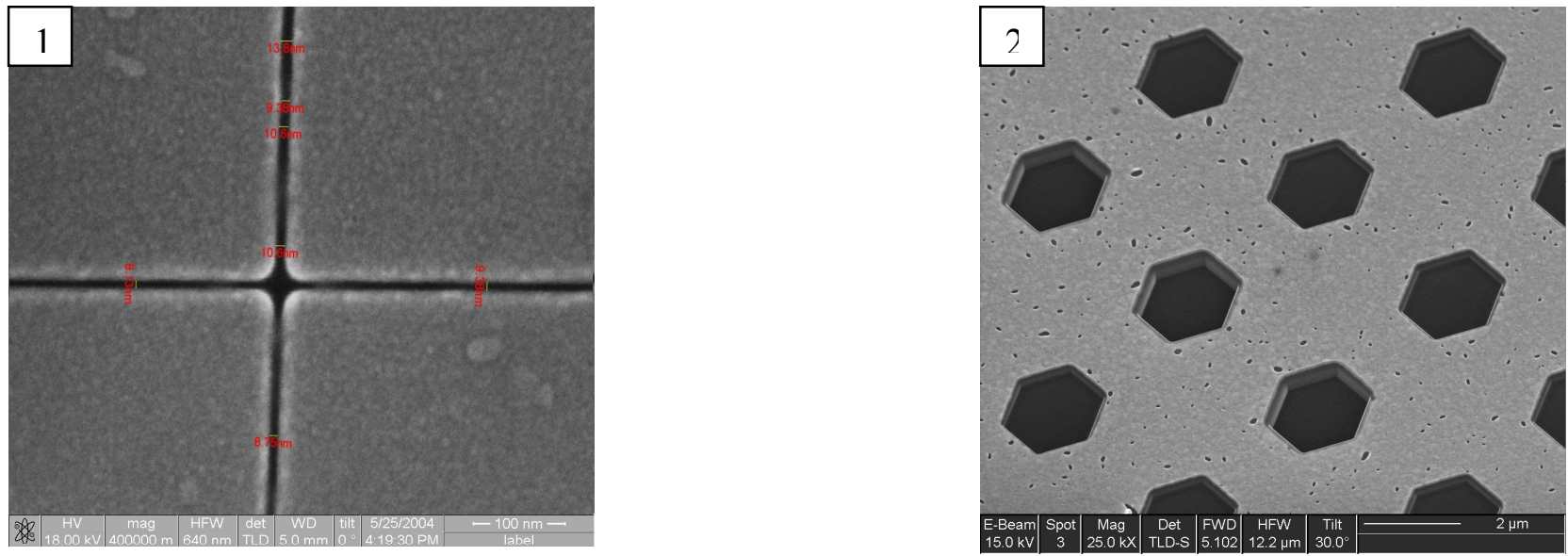

Fig 1. SEM image of minimum FIB mills of $\sim 10 \mathrm{~nm}$ made on a sample of chrome on glass.

Fig. 2. SEM image of photonic array produced in the DualBeam by FIB milling and incremental stage movements.

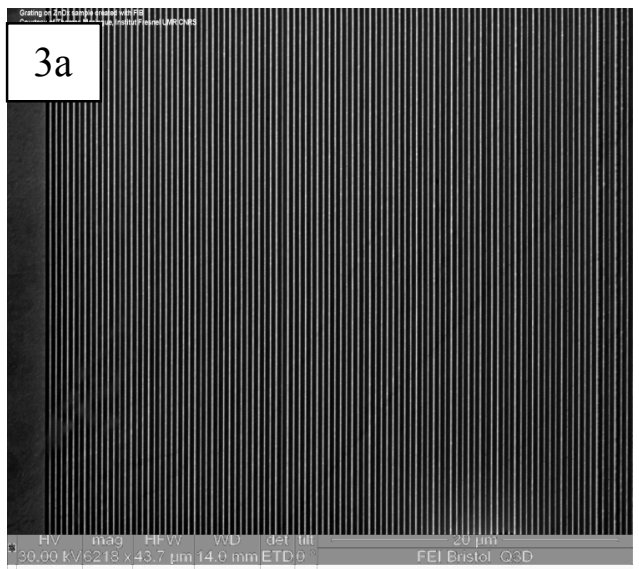

\section{$3 b$}

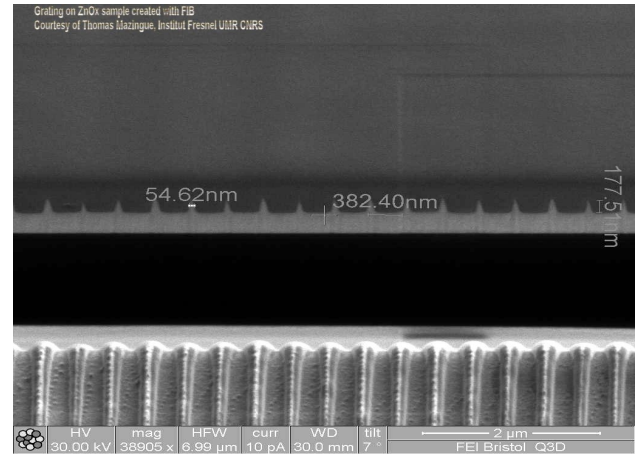

Fig 3a. SEM image of a diffraction grating milled in the DualBeam. The line spacing is achieved by incrementally changing the ion beam shift.

Fig. $3 \mathrm{~b}$. FIB image of cross section through the grating shown in fig $3 \mathrm{a}$.
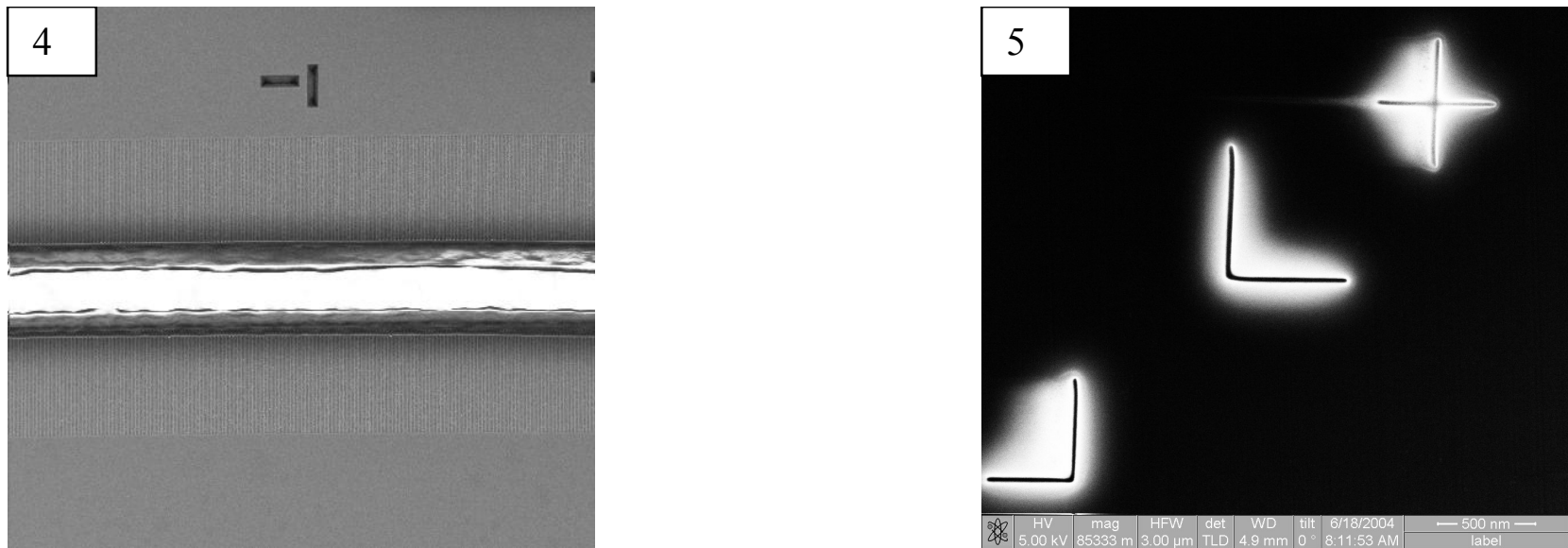

Fig 4. SEM image diffraction grating milled in the GaAs by the DualBeam using stage movement, beamshift and image recognition to accurately place milling patterns.

Fig. 5. SEM image of a series of patterns, which are the result of the DualBeam using the electron beam only to navigate to a point and accurately place a pattern for the ion beam to mill. 